\title{
Multi-focus Image Fusion Using Hybrid Transformation
}

\author{
Dr. Sheshang D. Degadwala ${ }^{* 1}$, Arpana Mahajan ${ }^{2}$, Dhairya Vyas ${ }^{3}$, Shivam Upadhyay ${ }^{4}$, Harsh S Dave \\ ${ }^{{ }_{1}}$ Head of Department, Computer Department, Sigma Institute of Engineering, Vadodara, Gujarat, India \\ ${ }^{2}$ Assistant Professor, Computer Department, Sigma Institute of Engineering, Vadodara, Gujarat, India \\ ${ }^{3}$ Assistant Professor, EC Department, Sigma Institute of Engineering, Vadodara, Gujarat, India \\ ${ }^{4}$ Assistant Professor, IT Department, Sigma Institute of Engineering, Vadodara, Gujarat, India \\ ${ }^{5}$ Medical Student, MBBS, Smt.B.K.Shah Medical Institute \& Research Centre, Gujarat, India
}

\begin{abstract}
The technique of blending two images or more than two images which produces outcome as the composite fused image. The obtained fused image is the upgraded version of original images because it has all the salient information. The present applications makes majority usage of this fused image to speed up their processing tasks in their respective fields. Recent real-time applications which require image fusion are remote sensing applications, medical applications, surveillance application, photography applications etc. the broad categorization of image fusion techniques are Non-transform domain or spatial domain and Transform domain or frequency domain. This paper initiates with the introduction of image fusion. In the second section it explains the analysis of multi-focus techniques. The third section explains hybrid image fusion strategy. Further sections elaborates the taxonomy of image fusion techniques and their comparative analysis with results.
\end{abstract}

Keywords : Multi-focus, Fusion, DWT, DRT

\section{INTRODUCTION}

The method of aggregating two images or aggregating additionally than two images which produces result as the blended or fused image. The acquired fused image is the upgraded version of source images because it has all the salient information. The present applications makes majority acceptance of this fused image to accelerate up their processing tasks in their corresponding fields. Recent real-time applications that makes use of the image fusion technique are remote sensing applications, medical applications, surveillance application and photography applications [13]. The afterward figure describes how image fusion can be applicable to several areas.
In Figure 1, every image (a) (b) has left blurred section and right blurred section respectively due to bad focus. This bad focus is because of the constraint of the optical lens i.e. it cannot capture all the objects in an image due to varying focal lengths. And, image is the after effect of the aggregation of two images by applying the image fusion technique. This illustration could be acclimated in a commercial advertisement photo shot. 


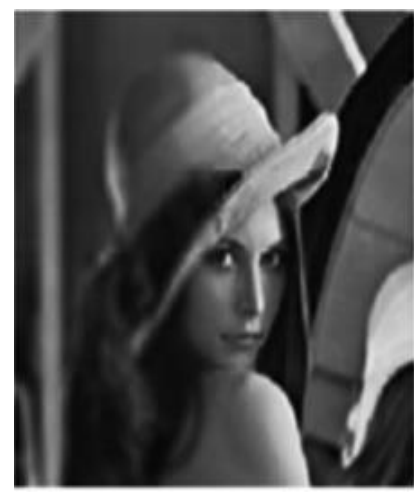

(a) Left blurred Image

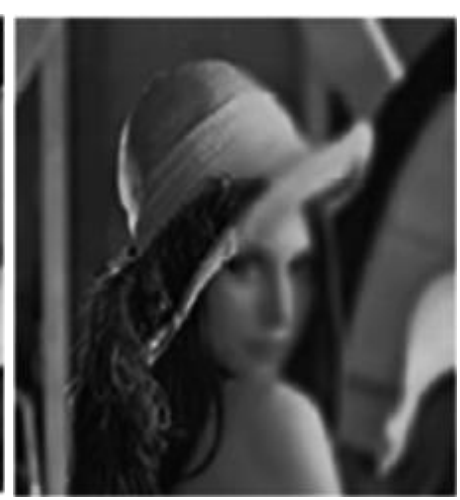

(b) Right blurred Image

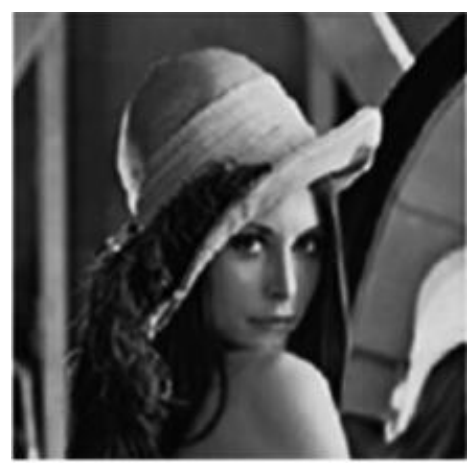

Figure 1: Example of Fused Image [10]

\section{MOTIVATION}

For the developing demand of the more clear as well as the majority of realistic data in the images that has gained attention to the number of researchers has helped to the improved image fusion algorithms. Image fusion subject is actually rapidly progresses from last couple of decades as it even now necessities up-gradation and improvement in the fusion technique that has been applied in varied recent many fields such as Remote-sensing, medical diagnosis, surveillance and photography applications. Image fusion can be characterized as the design of mixing two pictures alternately more than two pictures which will produce an absolute informative fused picture that might be in used for further examination procedure or those fused pictures could be further utilized within the above mentioned few recent realtime applications. Objective of the image fusion methodology is on to make images with very nearly fewer distortions and also towards maintaining the standards of the image in altogether aspects while analysing with the source or the original images.
I am focusing around issue in the photography field in which the imaging procedure includes taking a several shots with distinct camera settings, which will be very much time consuming. Therefore, how to incorporate those multi-focus image fusion algorithms under consumer electronics that will capture prominent fused image in real-time scenarios may be the inspiration for comprehending this challenging issue.

The proposed method that combines both the features i.e. Multi-resolution and Multi-scaling that are of the Transform Domain image fusion methodology. Multiresolution image Fusion technique is based on pixel level fusion methods. Wavelet Transform is the faster developed multi-resolution analysis image fusion method [5]. Multi-scaling transform is capable of representing images at different scales and different directions [4]. Curvelet, Ripplet, Contourlet Transform are multi-scaling geometric analysis image fusion methods [2].

\section{ANALYSIS OF MULTIFOCUS IMAGE FUSION TECHNIQUES}

In Non Transform domain fusion, the image's salient features are not clearly visible because of the following reasons:

It is affected by blurring effect which directly influences on the contrast of the image [29].

It causes color distortion [28].

Complex and time-consuming which are not suitable to be performed on real-time applications.

Due to the above limitations of the Spatial Domain or Non-transform methods, we have formulated the Research concept in the Transform Domain. There are many advancement in the different Transform methods, numerous sorts from the multi-resolution transforms to numerous others, for example, the Pyramid Decomposition, Discrete Wavelet Transform (DWT), Curvelet Transform (CVT), Contourlet Transform (CNT), Nonsubsampled Contourlet Transform (NSCT), Stationary Wavelet Transform (SWT) and Dual-tree intricate wavelet [19] etc. 
Additionally, over the Transform Domain another particular idea recently popular among researchers known as the Multi-scale Geometric Analysis (MGA) for high-dimensional signals have been developed. Furthermore, various MGA tools have been suggested such as the Ridgelet, Curvelet, Ripplet, Bandlet, Brushlet and also Contourlet transform [25].

The idea for Transform Domain fusion methodologies will be extensively used for image fusion nowadays and for this motivation behind we have carried out the Literature Review of Multi-focus Fusion methodologies inside the same Transform Domain. The underneath tables highlights those papers read about Multifocus image fusion which depicts about the Method used for fusion, its Key Characteristics, Merits and scope of improvement.

\section{PROBLEM DEFINITION}

For Multi-focus image fusion we need to blend the Discrete Wavelet Transform. Furthermore, the Discrete Ripplet Transform and also, in the last step of the method we apply the image blocking strategy. Multi-resolution image fusion technique are dependent upon the pixel level combination strategies whereas the Wavelet Transform is speedier formed
Multi-resolution analysis image fusion technique [14]. DWT provides for the exceptional as well as majority of the data towards distinct resolutions. Multi-scaling transform has been skilled about indicating to the images during different scales as well as the different directions [15]. Curvelet, Ripplet, Contourlet transforms have been the multi-scaling geometric analysis image fusion strategy [16]. DRT have been outlined to exemplify the images at distinct scales and also diverse directions. In the image blocking method, every block for the fused image has been matched and swapped for the first or the actual original block about any of the input source image and hence consequently enhances the visual aspect for the input images.

\section{PROPOSED SYSTEM}

The Figure 2 shows the Block Diagram of the last Block of the Proposed DWT + DRT method. In this first, the source images and the Initial Fused Image F1 are divided into Equi-Sized Blocks of size $\mathrm{m} \mathrm{x} \mathrm{n}$. In the next step, find out the Similarity values of the source input images. Lastly, the generation of Final Fused Image according to the chosen Highest Similarity value among calculated SM values for the source images.

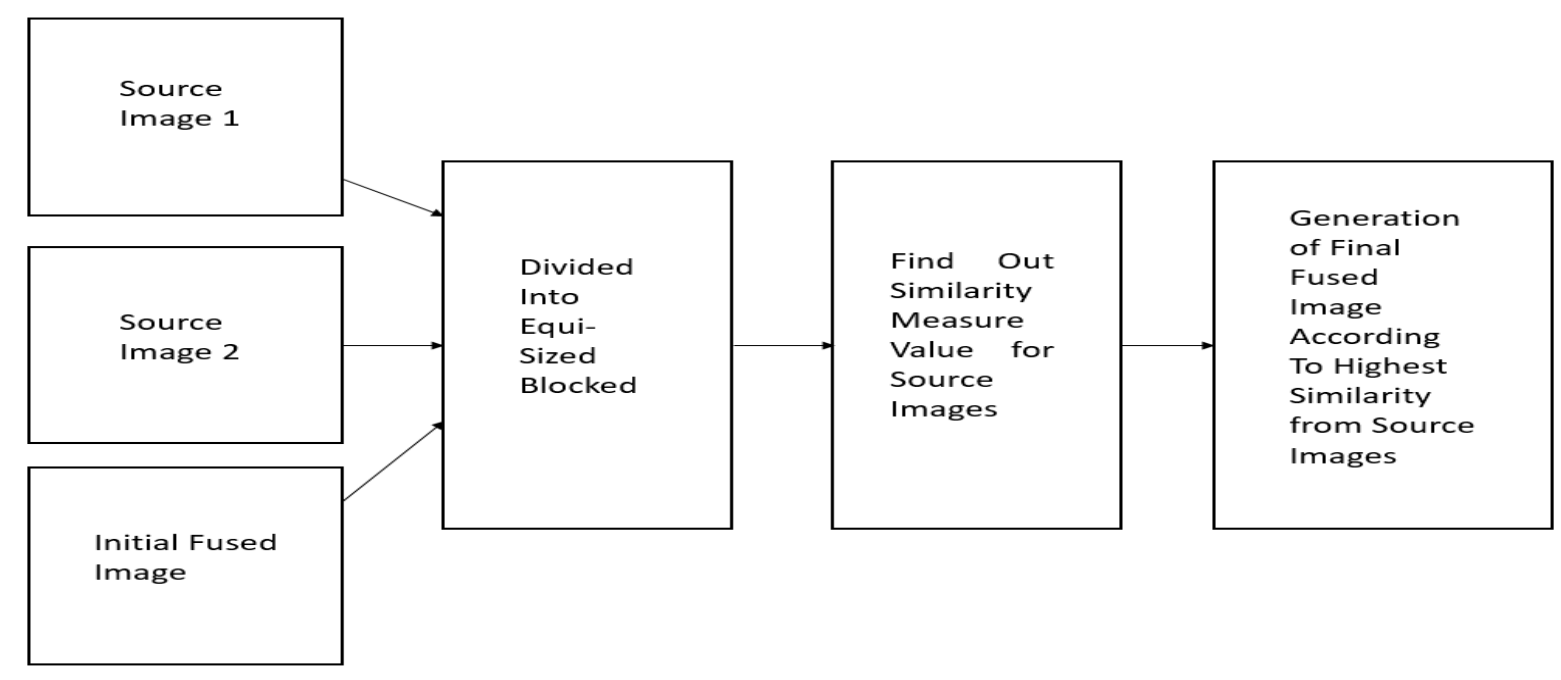

Figure 2: Block Diagram of Proposed Image Blocking Method

VI. Algorithm of Proposed System

Step 1 - Take the input Multi-focus images X1....Yn.
Step 2 - Convert the Color Multi-focus images to grayscale images. 
Step 3 - Normalize the input source images $\mathrm{x} 1$ to $\mathrm{x}$ and $y 1$ to $y$ respectively, so as to carry out the further calculations simpler. $\mathrm{X}=\mathrm{X} 1 / 255 ; \quad \mathrm{Y}=\mathrm{Y} 1 / 255$;

Step 4 - Apply DWT to the input images by performing single level DWT such that the images have been decomposed to four sub-bands i.e. one lowfrequency sub-band and three high-frequency subbands. (LL1, LH1, HL1, HH1) and (LL2, LH2, HL2, HH2) are the decomposed sub-bands of source images $\mathrm{X} 1$ and $\mathrm{Y} 1$ after DWT is applied.

Step 5 - LL1 and LL2 are the wavelet coefficients acquired from the input images after applying the DWT in the previous step 4. The low frequency coefficients gives high resolution and high spectral quality contents from the input images.

Step 6 - After getting the wavelet coefficients apply DRT to them to get the Ripplet coefficients DRX and DRY.
Step 7 - Find map.

Step 8 - Apply inverse DRT.

Step 9 - Apply inverse DWT.

Step 10 - Get the Initial Fused Image F1.

Step 11 - At this point apply the Image Blocking method. Here, partition the input images along with the initial fused image F1 under equi-sized square blocks whose size would be $\mathrm{m} \times \mathrm{n}$. Then calculate the similarity measure values(SM) of the relating sub blocks about input source images.

The greater the value of the SM indicates the high similarity between images. If the similarity between the input image block and the initial fused image block is more, the greater is the probability which implies that the output fused image blocks actually comes from the input image block.

Step 12 - Lastly, get the required final fused image.

\section{RESULT ANALYSIS}

Table 1: Comparative Analysis

\begin{tabular}{|l|l|l|l|l|l|l|l|l|}
\hline Dataset & $\begin{array}{c}\text { Existing } \\
- \text { RMSE }\end{array}$ & $\begin{array}{c}\text { Propose } \\
\mathrm{d}-\mathrm{RMSE}\end{array}$ & $\begin{array}{c}\text { Existing } \\
-\mathrm{MI}\end{array}$ & $\begin{array}{c}\text { Propose } \\
\mathrm{d}-\mathrm{MI}\end{array}$ & $\begin{array}{c}\text { Existing } \\
-\mathrm{Q}^{\mathrm{AB} / \mathrm{F}}\end{array}$ & $\begin{array}{c}\text { Propose } \\
\mathrm{d}-\mathrm{Q}^{\mathrm{AB} / \mathrm{F}}\end{array}$ & $\begin{array}{c}\text { Existing- } \\
\text { Execution } \\
\text { Time }\end{array}$ & $\begin{array}{c}\text { Proposed } \\
- \\
\text { Executio } \\
\mathrm{n} \text { Time }\end{array}$ \\
\hline Clock & 2.0504 & 2.1097 & 8.4205 & 9.852 & 0.79962 & 0.79962 & 12.3545 & 0.59669 \\
\hline Desk & 2.0904 & 1.2157 & 8.9649 & 9.8787 & 0.69966 & 0.8993 & 14.6108 & 0.59507 \\
\hline Doll & 2.0242 & 1.1584 & 9.8427 & 10.0181 & 0.79989 & 0.79966 & 24.2843 & 0.93119 \\
\hline Jug & 2.0109 & 2.1115 & 10.2328 & 11.1202 & 0.7999 & 0.89942 & 17.3735 & 0.67367 \\
\hline Lab & 1.0822 & 1.3563 & 8.8617 & 10.937 & 0.6997 & 0.89958 & 13.9285 & 0.56707 \\
\hline Pepsi & 2.0581 & 3.6725 & 7.7638 & 10.6814 & 0.69973 & 0.89953 & 10.403 & 1.3702 \\
\hline Rose & 2.1107 & 1.1126 & 10.1751 & 10.9013 & 0.79961 & 0.79951 & 22.7544 & 0.96762 \\
\hline
\end{tabular}




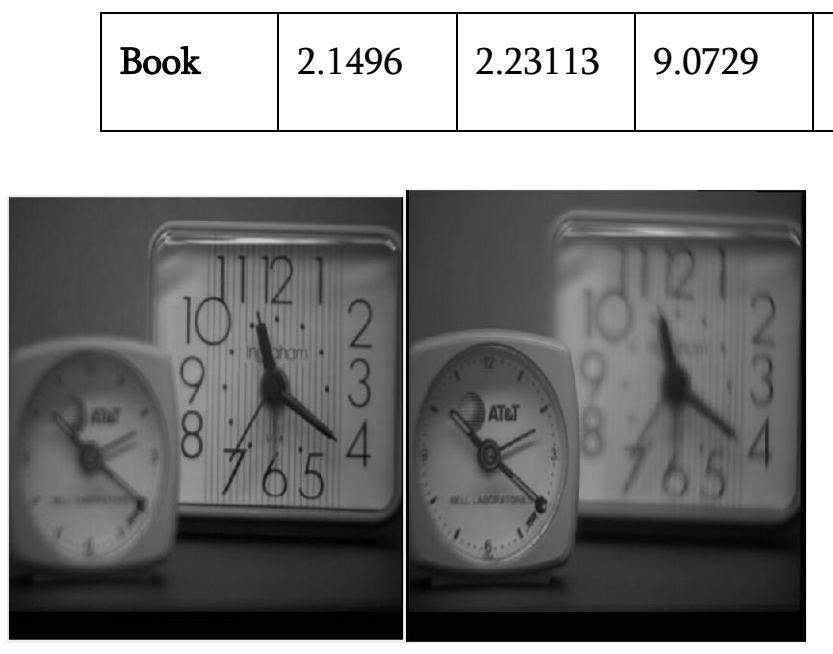

(a) Image $\mathrm{A}$

(b) Image B

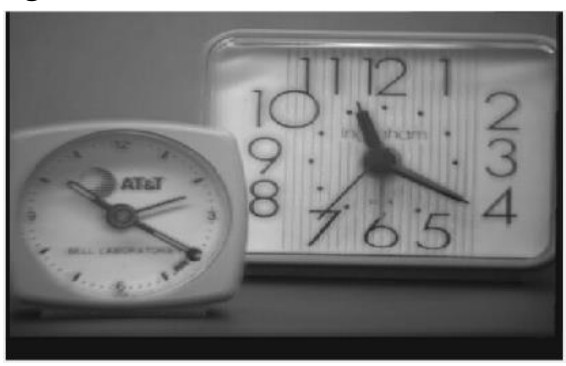

Figure 3: Fused DWT + DRT

The Figure 3 shows the Initial Fused Image Results of the Proposed Method for the Clock image.

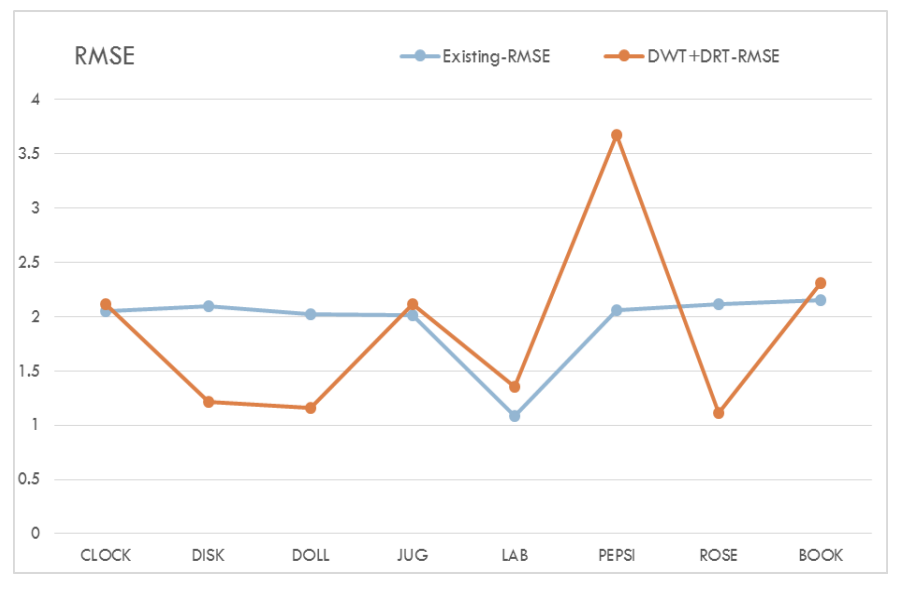

Figure 4: Fusion Result Analysis for RMSE

This section shows the above Result Analysis in the Chart form of the compared Existing FDCT based Image Fusion Method and the Proposed DWT + DRT up to the Initial Fused Image Block. The following Figure 4, Figure 5, Figure 6 and Figure 7 shows the chart of RMSE, MI, $Q^{A B / F}$ and Execution Time.

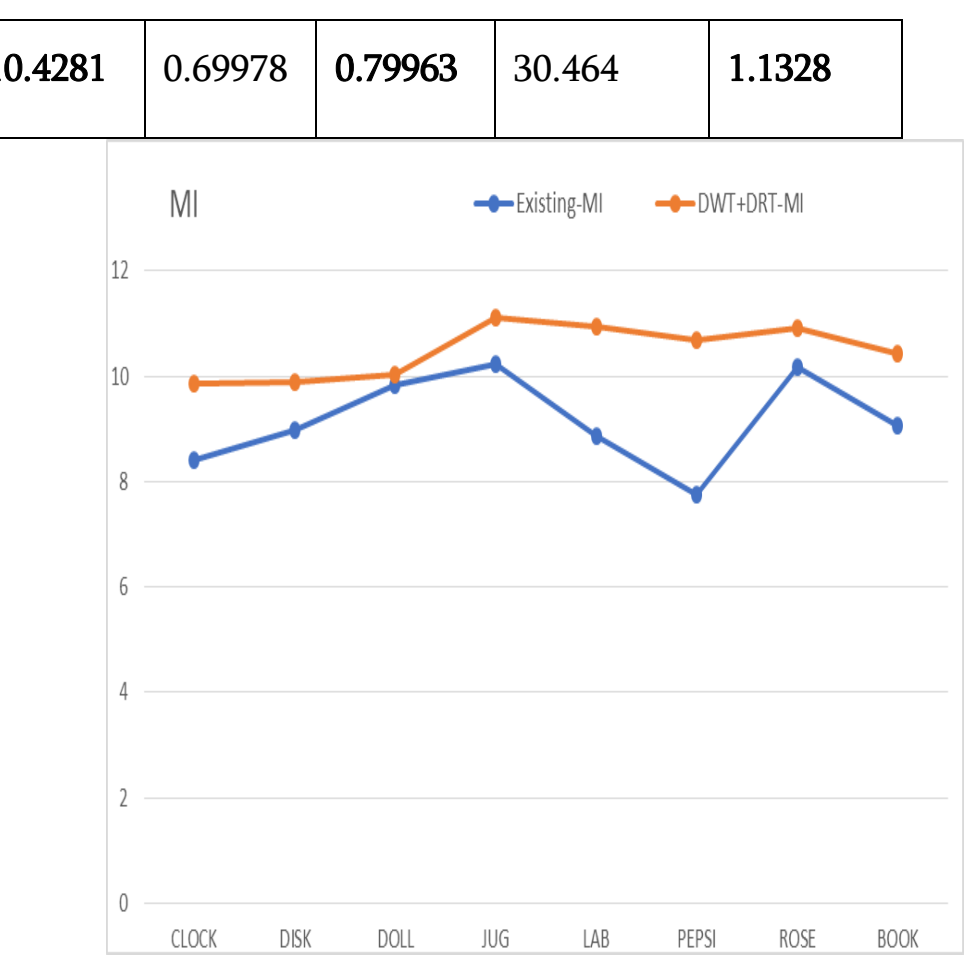

Figure 5: Fusion Result Analysis for MI

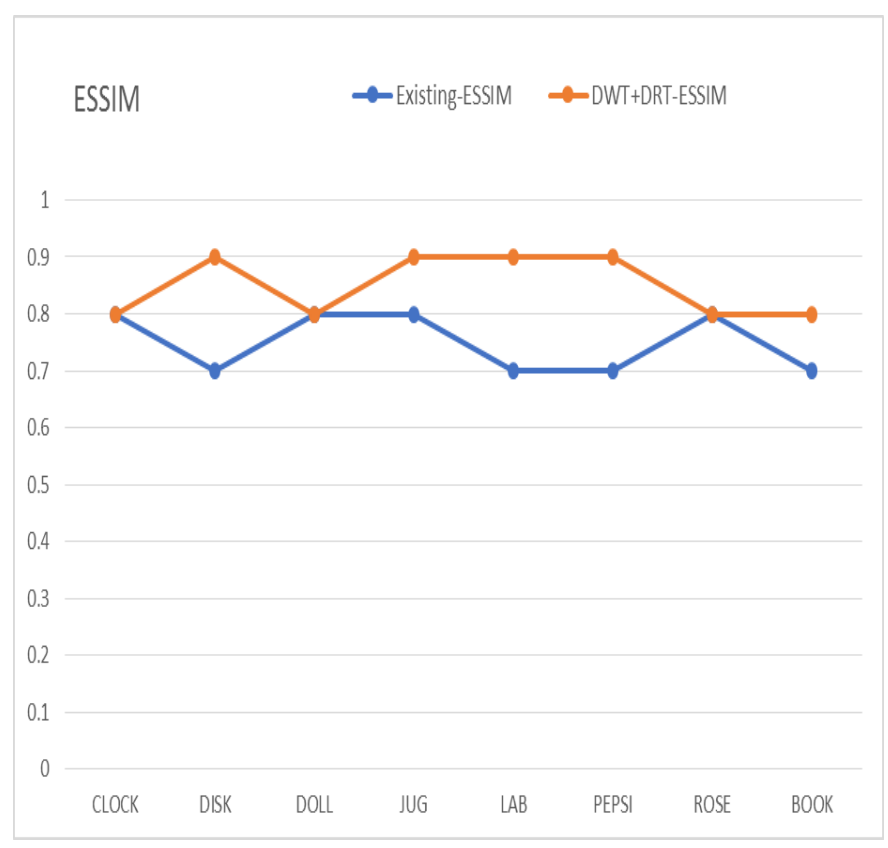

Figure 6: Fusion Result Analysis for $Q^{A B / F}$ 


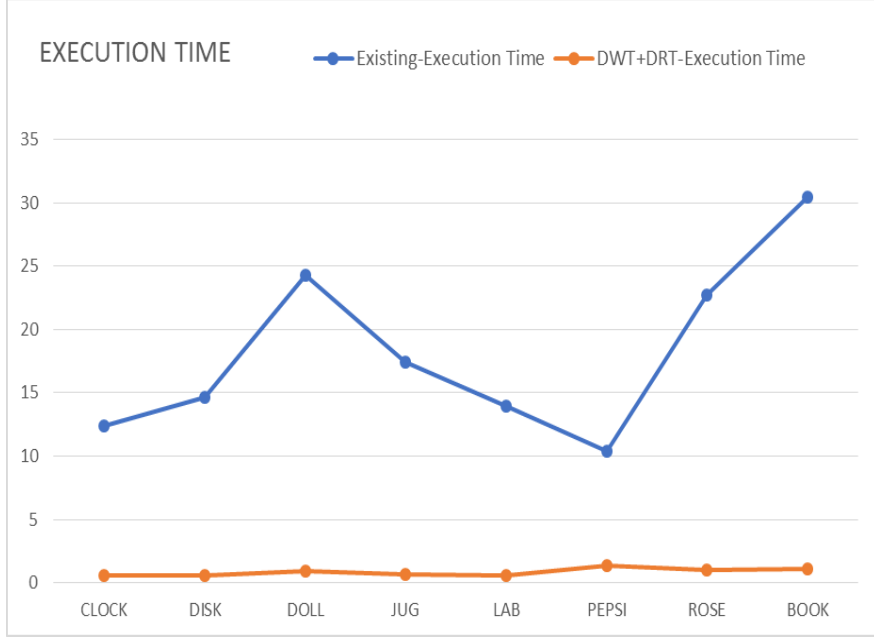

Figure 7: Fusion Result Analysis for Execution Time

\section{CONCLUSION}

Several methods are available for Multi-focus Image Fusion. All the spatial domain based methods have side effects like reducing the contrast of the entire image. But these are much useful and simpler in case of high contrast and bright images. The disadvantage of spatial domain approaches is that they produce spatial distortion in the fused image. Spatial distortion can be very well handled by frequency domain approaches on image fusion.

Limitation of DWT is that it does not provide directionality and anisotropy so it is not suitable for Multi-focus Image Fusion. Limitation of DWT can be overcome using DRT Transform. Also, the Limitation of DRT method is that it does not provide multiresolution. So, the limitation of DRT can be overcome using the DWT method. Hence, here Multi-focus Image Fusion is implemented using combination of DWT and DRT and we calculated the results up to the Initial Fused Image Block of the proposed method. The Experimental Results and Comparative Analysis can clearly states that the Proposed Fusion Framework gives superior results when compared to Existing FDCT based Fusion method.

In future the implementation of Proposed Image Fusion Method with Color images having more no. of input images. Further, will apply the image blocking method on the Initial Fused Image and will evaluate the Hybrid Multi-focus fusion approach based on evaluation metrics.

\section{REFERENCES}

[1]. Y. Yang, S. Tong, S. Huang, P. Lin and Y. Fang, "A Hybrid Method for Multi-focus Image Fusion Based on Fast Discrete Curvelet Transform", IEEE Access, pp. 1-1, 2017.

[2]. Aishwarya N, Abirami S and Amutha R, "Multifocus image fusion using Discrete Wavelet Transform and Sparse Representation", 2016 International Conference on Wireless Communications, Signal Processing and Networking (WiSPNET), 2016.

[3]. M. Dousty, S. Daneshvar and R. Sotero, "Multifocus image fusion via the Hartley transform", 2016 IEEE Canadian Conference on Electrical and Computer Engineering (CCECE), 2016.

[4]. M. Abdollahzadeh, T. Malekzadeh and $\mathrm{H}$. Seyedarabi, "Multi-focus image fusion for visual sensor networks", 2016 24th Iranian Conference on Electrical Engineering (ICEE), 2016.

[5]. K. Kalaivani and Y. Asnath Victy Phamila, "Analysis of Image Fusion Techniques based on Quality Assessment Metrics", Indian Journal of Science and Technology, vol. 9, no. 31, 2016.

[6]. Fuzhen Zhu, Aiping Jiang, Bing Zhu and Qun Ding, "Multifocus image fusion based on Uniform Discrete Curvelet Transform", 2015 International Conference on Estimation, Detection and Information Fusion (ICEDIF), 2015.

[7]. Paramanandham, N., Rajendiran, K., Narayanan, D., Indu Vadhani S. and Anand, M. (2015). An efficient multi transform based fusion for multi focus images. 2015 International Conference on Communications and Signal Processing (ICCSP).

[8]. M. Naji and A. Aghagolzadeh, "A new multifocus image fusion technique based on variance in DCT domain", 2015 2nd International Conference on Knowledge-Based Engineering and Innovation (KBEI), 2015. 
[9]. L. Cao, L. Jin, H. Tao, G. Li, Z. Zhuang and Y. Zhang, "Multi-Focus Image Fusion Based on Spatial Frequency in Discrete Cosine Transform Domain", IEEE Signal Processing Letters, vol. 22, no. 2, pp. 220-224, 2015.

[10]. Y. Phamila and R. Amutha, "Discrete Cosine Transform based fusion of multi-focus images for visual sensor networks", Signal Processing, vol. 95, pp. 161-170, 2014.

[11]. J. Tian and L. Chen, "Adaptive multi-focus image fusion using a wavelet-based statistical sharpness measure", Signal Processing, vol. 92, no. 9, pp. 2137-2146, 2012.

[12]. M. Haghighat, A. Aghagolzadeh and $H$. Seyedarabi, "Multi-focus image fusion for visual sensor networks in DCT domain", Computers \& Electrical Engineering, vol. 37, no. 5, pp. 789797, 2011.

[13]. S. Li, X. Kang, L. Fang, J. Hu and H. Yin, "Pixellevel image fusion: A survey of the state of the art", Information Fusion, vol. 33, pp. 100-112, 2017.

[14]. Wang Xin; Li Yingfang ; “A New Method For Multifocus Image Fusion Using Countourlet Transform", IEEE, 2011.

[15]. S. Das*,M. Chowdhury; M. K. Kundu; "Medical Image Fusion Based On Ripplet Transform Type-I", Progress In Electromagne -tics Research B, Vol. 30, 355-370, 2011.

[16]. Chandra Prakash; S Rajkumar; P.V.S.S.R. Chandra Mouli; "Medical Image Fusion Based on Redundancy DWT and Mamdani Type Minsum Mean-of-max Techniques with Quantitative Analysis", IEEE, 2012.

[17]. Madhuri J. Patel; Keyur N. Brahmbhatt; Z. H. Shah; "Comparative Study on Image Fusion Methods", International Journal of Graphics \& Image Processing, Vol.4,issue 1,Feb. 2014.

[18]. Wei-Wei Wang, Peng-Lang Shui and GuoXiang Song, "Multifocus image fusion in wavelet domain", Proceedings of the 2003 International Conference on Machine Learning and Cybernetics (IEEE Cat. No.03EX693).
[19]. J. Xu, L. Yang and D. Wu, "Ripplet: A new transform for image processing", Journal of Visual Communication and Image Representation, vol. 21, no. 7, pp. 627-639, 2010.

[20]. What's New - MATLAB", In.mathworks.com, 2016. Online]. Available: https://in.mathworks.com/products/matlab/what snew.html. Accessed: 29- Nov-2016].

[21]. OpenCV: Introduction", Docs.opencv.org, 2016.Online].Available:http://docs.opencv.org/3. 1.0/d1/dfb/intro.html. Accessed: 01 - Dec2016].

[22]. A. Noskov and A. Priorov, "Comparison of image Quality Assessment Methods for multiFocused Image Fusion", Proceedings of the 20th Conference of Fruct Association, 2015.

[23]. S. Li and B. Yang, "Multifocus image fusion by combining curvelet and wavelet transform", Pattern Recognition Letters, vol. 29, no. 9, pp. 1295-1301, 2008.

[24]. S. Das*, M. Chowdhury; M. K. Kundu; "Medical Image Fusion Based On Ripplet Transform Type-I", Progress In Electromagnetics Research B, Vol. 30, 355- 370, 2011.

[25]. U. Patil and U. Mudengudi, "Image fusion using hierarchical PCA.", International Conference on Image Information Processing, 2011.

[26]. R.A. Mandhare, P. Upadhyay and S.Gupta, "Pixel-level image fusion using Brovey Transform and Wavelet Transform", International Journal of Advanced Research on Electrical, Electronics and Instrumentation Engineering, Vol. 2, Issue 6, June 2013.

[27]. M. Choi, "Erratum to "A new look at IHS-like image fusion methods" Information Fusion 2 (2001) 177-186]", Information Fusion, vol. 8, no. 2, p. 217, 2007.

[28]. G. Piella and H. Heijmans, "A new quality metric for image fusion", Proceedings 2003 International Conference on Image Processing (Cat. No.03CH37429).

[29]. Gehad Mohamed Taher, Mohamed ElSayed Wahed and Ghada EL Taweal,"New Approach 
for Image Fusion Based on Curvelet Approach",International Journal of Advanced Computer Science and Applications, Vol. 5, No. 7, 2014.

[30]. C. K. Solanki and N. M. Patel, "Pixel based and Wavelet based Image fusion Methods with their Comparative Study", National Conference on Recent Trends in Engineering \& Technology, Pg no.13-14, May 2011.

[31]. A. Malviya and S. G. Bhirud, " Image Fusion of Digital Images", International Journal of Recent Trends in Engineering, Vol 2, No. 3, November 2009.

[32]. M. Nejati, S. Samavi, S. Shirani, "Multi-focus Image Fusion Using Dictionary-Based Sparse Representation", Information Fusion, vol. 25, Sept. 2015, pp. 72-84.

[33]. A. Moonon and J. Hu, "Multi-Focus Image Fusion Based on NSCT and NSST", Sensing and Imaging, vol. 16, no. 1, 2015.

[34]. Lytro Multi-focus Image Dataset. Available from:https://www.researchgate.net/publication/ 291522937_Lytro_Multi-focus_Image_Dataset accessed Oct 06 2017].

[35]. "MATLAB GUI", In.mathworks.com, 2018. Online].Available:https://in.mathworks.com/dis covery/matlab-gui.html. Accessed: 04- Mar2018].

[36]. "Cite a Website - Cite This For Me", Ece.uwaterloo.ca, 2018. Online]. Available: https://ece.uwaterloo.ca/ $\sim$ nnikvand/Coderep/gui \%20examples/GUI.pdf. Accessed: 04- Mar2018]. 